\title{
Sensors and Actuators using Ionic Polymer Metal Composites for Micro Robot Applications
}

\author{
B.G.L.TSamaranayake
}

\begin{abstract}
As the name implies, Ionic Polymer Metal Composite (IPMC) is a composite material, which deforms when exposed to an electric field. On the other hand it is capable of generating a voltage across the top and the bottom surfaces of the material, when subjected to mechanical deformations. Therefore IPMCs can be used as Soft Actuators as well as Soft Sensors. Mechanical flexibility and light-weight, make them 'Soft'. However, designing Soft Sensors using IPMCs requires accurate input-output models from the mechanical input to the electrical output. Similarly for Soft Actuator designs, models have to be derived from the electrical input to the mechanical output.
\end{abstract}

This paper uses cantilevered flexible beam model undergoing large deflections under a point load at the free end to model an IPMC Soft Sensor. The same cantilevered flexible beam model but under a uniformly distributed load undergoing large deflections is used to model an IPMC Soft Actuator. The principle of thermal expansion and contraction of a bi-metallic strip is used to model the motion action in both cases.

The structural and thermal multi-physics models, used to derive the deformations of complicated shapes of actuators are implemented for simulations using finite element methods. The simulations are verified experimentally using IPMCs manufactured locally.

Keywords: Ionic Polymer Metal Composite, Soft Actuators, Soft Sensors

\section{Introduction}

Thanks to the advancements in Chemistry and Material Sciences, sensors and actuators made of soft material, hereafter called as Soft Sensors and Soft Actuators, are in a position to simply replace electromechanical sensors and low load actuators used mainly in micro robotic applications ranging from biomedical engineering to the novel concept of unmanned solid-state aircrafts [1]. Due to the ability to work in free air, Ionic Polymer Metal Composites (IPMC) becomes a prominent material for soft sensors and soft actuators.

IPMC consist of a thin polyelectrolyte membrane of Nafion, Flemion, or Aciplex, plated on both faces by a noble metal, generally platinum or gold or platinum with a layer of finishing gold to improve surface conductivity. It is neutralized with the necessary amount of counter-ions such as $\mathrm{H}^{+}, \mathrm{Li}^{+}, \mathrm{Na}^{+}, \mathrm{K}^{+}$etc, balancing the charge of anions covalently fixed to the backbone membrane [2]. Despite the complicated chemical structure and lack of published literature on the fabrication process, the authors of the paper have derived their own, low cost fabrication technology, which is of supreme importance in promoting IPMCs for micro robot applications and other research activities for a developing country like Sri Lanka.

When a thin strip of an IPMC membrane in the hydrated state is stimulated by the application of a small (1 to $3 \mathrm{~V}$ ) alternating potential, it undergoes a bending vibration at the frequency of the applied voltage, generally no more than a few Hertz. Under a suddenly applied step voltage, the composite quickly bends towards the anode. Hence IPMCs can be used as voltage operated Soft Actuators. The bending scenario under a constant voltage is shown in Fig. 01. In the absence of an applied voltage, the cations and anions form conjugate pairs, with the cations optimally distributed within each cluster within the membrane to minimize the overall energy as shown in Fig.01 (a). Each cation-anion pair may be visualized as a pseudo-dipole whose

Eng. (Dr.) B. G.L.T. Samaranayake, AMIE(SL), MIET(London), MIEEE(USA),B.Sc. Eng. (Hons) (Peradeniya), Tech. Lic. (KTH, Sweden), Ph.D (KTH,Sweder), Senior Lecturer, Department of Electrical and Electronic Engineering, Faculty of Engineering, University of Peradeniya, Sri Lanka. 
effective moment depends on the nature of the cation and the number of available molecules.

An applied electric field affects the cation distribution within the membrane, forcing the cations to migrate towards the cathode. This change in the cation distribution produces two thin layers, one near the anode and another near the cathode boundaries. These two layers are referred as anode and cathode boundary layers, respectively. The clusters within the anode boundary layer are depleted of their cations, while clusters within the cathode boundary layer are cation rich as shown in Fig.01 (b).

At the anode and cathode boundary layers, the respective reduction and increase in the concentration of the ions may produce any of the following effects:

1) A decrease in the effective stiffness of the polymer in the anode, and an increase in this stiffness in the cathode boundary layer, respectively.

2) A repulsive electrostatic force among the fixed anions of each cation-depleted cluster in the anode boundary layer, which tends to increase the average cluster volume and also relax the pre-stretched polymer chains between adjacent clusters, increasing their entropy and decreasing their elastic energy. In the clusters within the cathode boundary layer, the additional cations initially repel the existing cation-anion pseudodipoles, contributing to the rapid expansion of these clusters and hence the fast bending motion of the IPMC towards the anode as shown in Fig. 01 (c).

The magnitude and speed of these deflections depend on the nature of the counter-ions, the structure of the electrodes, the level of hydration (solvent saturation), etc. When the same membrane is suddenly bent, a small voltage of the order of millivolts is produced across its faces. Hence, the IPMCs can be used as Soft Sensors as well.

\section{Shape Modelling}

This section describes the analytical modelling of soft actuators and soft sensors with the intension of obtaining the deformed shape.

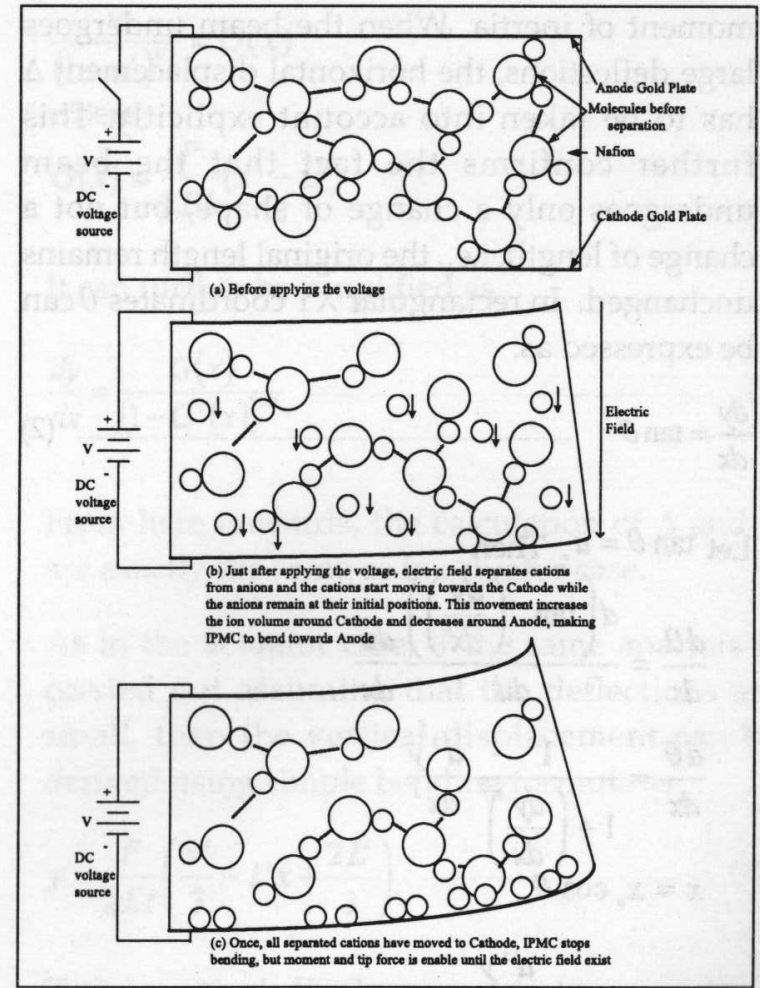

Figure 1: The bending scenario of an IPMC under a constant voltage

\subsection{Soft Actuator Deformed Shape Model}

The axial stresses are induced by the normal stresses generated due to the cation distribution explained and high Poisson ratio of about 0.33 . The normal stresses remain perpendicular to the electrode surface of the beam, irrespective of the shape deformation it undergoes. Therefore, with the absolute maximum voltage the beam ends up in a circular shape [3]. Hence, for modeling purpose, it can be considered that the beam is under a uniformly distributed external load as shown in Fig. 02(a). The equilibrium position at steady state is reached, when the external stresses and moments neutralize the internally induced stresses and moments.

The Euler-Bernoulli law [4] states that the bending moment $M$ is proportional to the change in the curvature produced by the action of the load. This law may be written mathematically as follows:

$\frac{1}{R}=\frac{d \theta}{d x_{0}}=\frac{M}{E I}$

where $\mathrm{R}$ is the radius of curvature, (is the slope at any point $x_{\sigma}$ where $x_{0}$ is measured along the arc length as shown in Fig. 02(b), $E$ is the modulus of elasticity, and I is the cross-sectional 
moment of inertia. When the beam undergoes large deflections, the horizontal displacement $\Delta$ has to be taken into account explicitly. This further confirms the fact that the beam undergoes only a change of shape, but not a change of length. i.e., the original length remains unchanged. In rectangular $\mathrm{XY}$ coordinates $\theta$ can be expressed as:

$$
\frac{d y}{d x}=\tan \theta
$$

$$
\begin{aligned}
& \text { Let } \tan \theta=u \text {. Then } \\
& \frac{d \theta}{d x}=\frac{d\left(\tan ^{-1}\left(\frac{d y}{d x}\right)\right)}{d u} \frac{d u}{d x} \\
& \frac{d \theta}{d x}=\frac{1}{1+\left(\frac{d y}{d x}\right)^{2}} \frac{d^{2} y}{d x^{2}} \\
& x=x_{0} \cos \theta \\
& \frac{d \theta}{d x_{0}}=\frac{\frac{d^{2} y}{d x^{2}}}{\left(1+\left(\frac{d y}{d x}\right)^{2}\right)^{3 / 2}} \\
& \frac{d \theta}{d x_{0}}=\frac{\frac{d u}{d x}}{\left(1+u^{2}\right)^{3 / 2}}
\end{aligned}
$$

Since the distributed force act perpendicular to the beam surface, the moment at a distance $x_{0}$ along the length of the beam becomes

$M=\frac{w x_{0}^{2}}{2}=\frac{w x^{2}}{2 \cos ^{2} \theta}=\frac{w x^{2}\left(1+u^{2}\right)}{2}$.

By substituting for $M$ and $d \theta / d x_{0}$ in the EulerBernoulli law in equation (1), the following relation can be obtained [4]:

$\int \frac{d u}{\left(1+u^{2}\right)^{3 / 2}}=\int \frac{w x^{2}}{2 E I} d x$

Solving the integration by substitution and using the trigonometric identity

$\cos 3 \theta=3 \cos \theta-4 \cos ^{3} \theta$ gives

$\frac{u\left(3+2 u^{2}\right)}{\left(1+u^{2}\right)^{1 / 2}}=G(x)$

Applying boundary conditions for the cantilevered beam results in

$G(x)=\frac{w}{6 E I}\left(x^{3}-L_{0}^{3}\right)$

where $L_{\mathrm{o}}=L-\Delta$. where $L_{0}=L-\Delta$.

It can further be simplified as

$\frac{d y}{d x}=\sqrt{\frac{\left(1+\left(\frac{2 G(x)}{3}\right)^{2}\right)^{1 / 2}-1}{2}}$

The large deflection at any $0 \leq x \leq L_{0}$ may now be obtained by integrating $d y / d x$ once and satisfying the boundary conditions at $x=L_{0}$. However, since $L_{0}=L-\Delta, \Delta$, must be known first. Since the beam is inextensible, the deformed length must be equal to the original length. Therefore

$L=l_{0}^{4}\left(1+\left(\frac{d y}{d x}\right)^{2}\right)^{1 / 2} d x$

This relation can be used in the procedure given below to find $\Delta$ by trial and error.

1. Assume a value for $\Delta$ such that $0 \leq \Delta \leq L$

2. Calculate $G(x)$ from equation (3).

3. Find $d y / d x$ from equation (4).

4. Calculate $L$ from equation (5) by solving the integration numerically, and check if it matches with the original $L$.

5. Repeat the steps $1,2,3$ and 4 until the calculated $L$ matches the original beam length $L$.

Once $\Delta$ has been calculated correctly, $y$ can be obtained by numerically solving the integration in equation (4). If the same analysis is carried out assuming that the deflections are small, then no horizontal displacement is accounted, i.e., $\Delta=0$. Further the vertical displacement can be derived using simple bending formula as

$y=\frac{w}{6 E I}\left(\frac{x^{4}}{4}-L^{3} x+\frac{3 L^{4}}{4}\right)$

where the distance is measured from the free end of the beam.

\subsection{Soft Sensor Deformed Shape Model}

The soft actuators show planer deflections due to chemo-mechanical internal forces caused by an externally applied electro motive force. Considering the reverse process of this, if a planer deflection is caused by means of an external force, an electro motive force is generated. Therefore it can be considered as a 


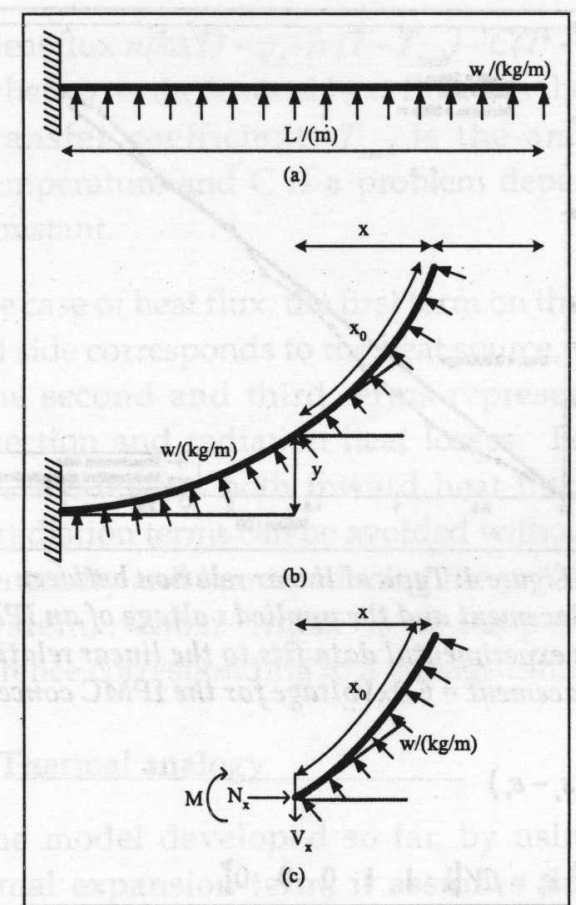

Figure 2: (a). The flexible beam is under a uniformly distributed force. (b). Internally generated distributed forces remaining perpendicular to the surface. (c). A sectioned portion of the flexible beam of length $x_{0}$ measured along the beam and $x$ is measured from the beam tip.

sensor. The generated voltage can be used to detect the deflected location. These defections in general, are large compared to the dimensions of the flexible beam. Therefore large deflection theory has to be followed in deriving the equations representing the planer displacements. Since the force is applied by an external body, it is more natural to represent it as a point force applied at the beam tip as shown in Fig. 03. Otherwise, if the soft sensor is sandwiched with the soft actuator, it can be considered as a uniformly distributed force, where the analysis resembles the previous section. Here too the analysis follows the same steps as that for the soft actuator case. However, the moment $\mathrm{M}$ is different for point force as

$M=P x$

Similar to the actuator case, by substituting for $\mathrm{M}$ and $d \theta / d x_{0}$ in the Euler-Bernoulli law in equation (1), the following relation can be obtained:

$\int \frac{d u}{\left(1+u^{2}\right)^{3 / 2}}=\int \frac{P x}{E I} d x$

Solving the integration by substitution and applying boundary conditions gives $\frac{u}{\left(1+u^{2}\right)^{1 / 2}}=G(x)$

where

$G(x)=\frac{P}{2 E I}\left(x^{2}-L_{0}^{2}\right)$

It can further be simplified as

$\frac{d y}{d x}=\frac{G(x)}{\left(1-G^{2}(x)\right)^{1 / 2}}$

From here onwards, the calculation of $\Delta$ and y are exactly the same as in actuator case.

As in the actuator case, if the same analysis is carried out assuming that the deflections are small, then the vertical displacement can be derived using simple bending formula as

$y=\frac{P}{2 E I}\left(\frac{x^{3}}{3}-L^{2} x+\frac{2 L^{3}}{3}\right)$

If the vertical displacement is large under a considerably large force, the beam will have to be considered extended, to match its original dimensions. Even under the same force as in the large deflection case, the magnitude of the vertical displacement given by equation (10) can be much larger than that from equation (9). Because equation (10) always considers $\Delta=0$ condition and assumes that the displaced beam

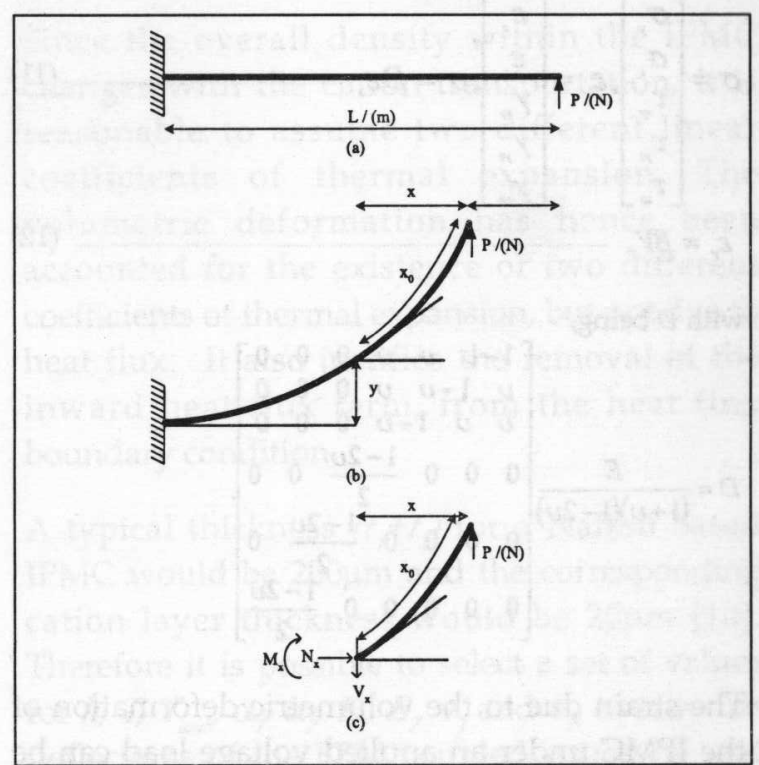

Figure 3: (a). The flexible beam is under a point force exerted from externally. (b). Since the point force is externally, it is assumed to remain vertically, while the beam deflects. (c). A sectioned portion of the flexible beam of length $x_{0}$ measured along the beam and $x$ is measured from the beam tip. 
tip will be vertically above the beam tip. However, this is not the reality. Therefore, the equations derived under large deflection theory must be used in deciding the shape of the beam under a tip or a uniformly distributed force.

\section{Finite element analysis}

This section describes the theory behind the coupled structural and thermal multi-physics model used to describe the deformation action.

Since the deformation scenario is common for both soft sensor case and soft actuator case, they both are covered together.

\subsection{Mathematical derivation}

In the theoretical analysis of structures, the elasticity phenomena is governed by the Hooke's law expressed in three dimensions by equation (11), where the strain $\varepsilon_{s}$ and the stress $\sigma$ in the body are related by the elasticity matrix $D[5] . E$ and $v$ are the modulus of elasticity (Young's modulus) and Poisson's ratio respectively.

It has been shown both in previous studies [6][7][8] and here (Fig. 04) that the IPMCs develop the strains depending on the applied voltage, whose relations can be derived to be linear as

$$
\sigma=\left[\begin{array}{l}
\sigma_{x} \\
\sigma_{y} \\
\sigma_{z} \\
\tau_{x y} \\
\tau_{x z} \\
\tau_{x}
\end{array}\right], \varepsilon_{,}=\left[\begin{array}{l}
\varepsilon_{x} \\
\varepsilon_{y} \\
\varepsilon_{z} \\
\gamma_{x y} \\
\gamma_{y z} \\
\gamma_{x x}
\end{array}\right], \sigma=D \varepsilon,
$$

$$
\varepsilon_{v}=\beta V \text {. }
$$

$$
\begin{aligned}
& \text { with } D \text { being, }\left[\begin{array}{ccccccc}
1-v & v & v & 0 & 0 & 0 \\
v & 1-v & v & 0 & 0 & 0 \\
v & v & 1-v & 0 & 0 & 0 \\
0 & 0 & 0 & \frac{1-2 v}{2} & 0 & 0 \\
0 & 0 & 0 & 0 & \frac{1-2 v}{2} & 0 \\
0 & 0 & 0 & 0 & 0 & \frac{1-2 v}{2}
\end{array}\right]
\end{aligned}
$$

The strain due to the volumetric deformation of the IPMC under an applied voltage load can be considered as an incident without shearing [9]. In order to take into account the interaction between the mechanical behaviour and the actuation principle, the elastic equation of the actuator under an applied voltage can be expressed as:

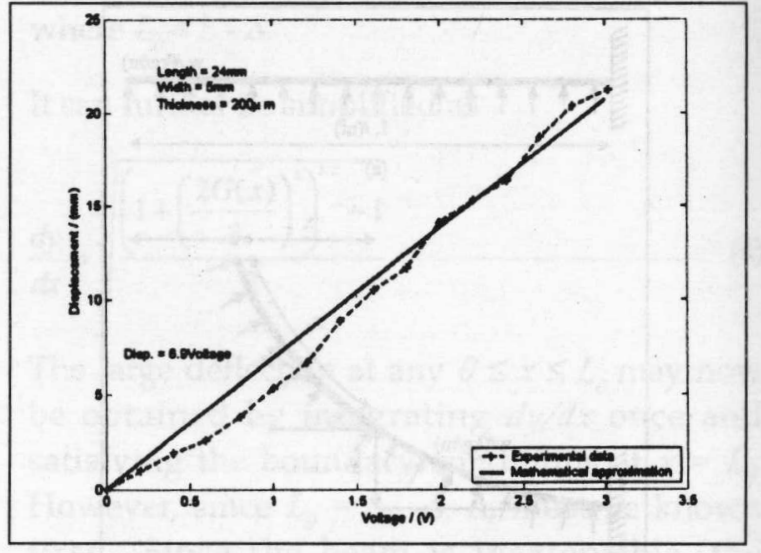

Figure 4: Typical linear relation between displacement and the applied voltage of an IPMC. The experimental data fits to the linear relation Displacement $=6.9 \times$ Voltage for the IPMC concerned .

$\sigma=D\left(\varepsilon_{1}-\varepsilon_{\nu}\right)$

where $\varepsilon_{r}=\beta V\left[\begin{array}{llllll}1 & 1 & 1 & 0 & 0 & 0\end{array}\right]^{\top}$

A finite element method (FEM) software is used to simulate the mathematical model described by equation (13). However since this is a novel concept, the standard FEM software do not support this particular equation. Nevertheless, many of them include coupled-field analysis (multi-physics) based on the theory of thermoelasticity. There the analysis takes into account the interaction between the mechanical and thermal fields and uses the following equation:

$\sigma=D\left(\varepsilon_{s}-\varepsilon_{p}\right)$

where $\varepsilon_{T}=\alpha T\left[\begin{array}{llllll}1 & 1 & 0 & 0 & 0\end{array}\right]^{T}$

Further, $T(x, y, z)$ is the non-uniform temperature field in the body and $\alpha$ is the coefficient of thermal expansion. For the purpose of modelling, the thermo-elasticity equation can be used if the voltage is mimicked by the temperature $T$, and the coefficient of thermal expansion by the slope $\beta$ of the strainvoltage linear approximation, i.e., $V \rightarrow T \rightarrow \beta \rightarrow a$.

In the thermo-elasticity model, the surface boundary conditions can be defined as one of the following:

a). Constant temperature $T=T_{0}$

b). Thermal insulation $n(k \Delta T)=0$ where $n$ is the vector normal to the surface of the body and $\mathrm{k}$ is the thermal conductivity 
c). Heat flux $n(k \Delta T)=q_{0}-h\left(T-T_{a m b}\right)-C\left(T^{4}-T^{4 a m b}\right)$, where $q_{0}$ is the inward heat flux, $h$ is the heat transfer coefficient, $T_{a m b}$ is the ambient temperature and $\mathrm{C}$ is a problem dependant constant.

In the case of heat flux, the first term on the right hand side corresponds to the heat source, where as the second and third terms represent the convection and radiation heat losses. For the IPMC modelling, both inward heat flux term and radiation terms can be avoided without loss of generality and hence reducing the problem to a system, whose input is a temperature difference corresponding to a voltage difference.

\subsection{Thermal analogy}

In the model developed so far, by using the thermal expansion term, it assumes that the overall volume of the IPMC changes. However, in reality, it remains unchanged and only the shape change takes place due to the cation transportation. In order to incorporate this in the model, the same thermal model is used in a bit different manner so as to keep the overall volume constant and to count only for the change of the shape. This is accommodated in the FEM model by introducing the bi-metallic strip structure into the model. Two regions in the cross section have been identified as the Cation vacating area and Cation accumulating area caused by the cation transportation as shown in Fig. 05.

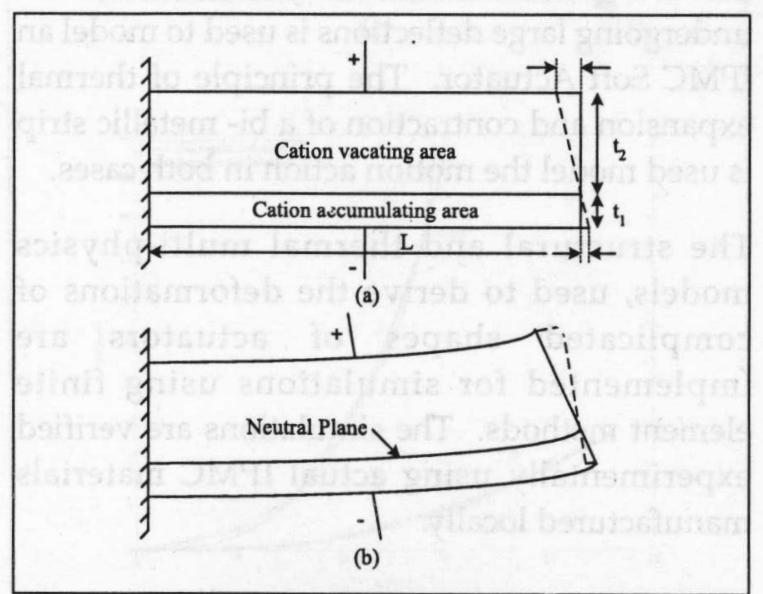

Figure 5(a): With the applied voltage, two layers can be identified as cation accumulating area and cation vacating area, (b) modelling the volumetric change caused by the cation transportation due to an applied voltage is as same as a deformation of a bi-metallic strip due to a heat flow caused by a temperature difference.
For the modelling purpose, the temperature of the Cation accumulated area is assumed to have increased to $T_{a m b}+T_{1}$ from $T_{a m b}$ and it is reduced to $T_{a m b}-T_{2}$ from $T_{a m b}$ in the other area. Further it assumes that the neutral plane temperature remains unchanged at $T_{a m b}$ and the deformation takes place about that plane, corresponding to the neutral plane of the actual bending (not the horizontal symmetric plane).

For the original volume to remain unchanged, the cation accumulating area and the cation vacating area should follow the following relation:

$L\left(t_{1}+t_{2}\right)=\left(L+\frac{\delta_{1}}{2}\right) t_{1}+\left(L-\frac{\delta_{2}}{2}\right) t_{2}$.

By defining the linear coefficient of thermal expansion for the two regions as ( 1 and $(2$, the thermal expansion and contraction due to the temperature rise $T_{a m b}+T_{1}$ and drop $T_{a m b}-T_{2}$ in the respective areas become:

$\frac{\delta_{1}}{L}=4 \alpha_{1} T_{1}$
$\frac{\delta_{2}}{L}=-4 \alpha_{2} T_{2}$

By simplifying equations (15), (16) and (17), the following relation can be obtained:

$\frac{t_{2}}{t_{1}}+\frac{\alpha_{1} T_{1}}{\alpha_{2} T_{2}}=0$

Since the overall density within the IPMC changes with the cation transportation, it is reasonable to assume two different linear coefficients of thermal expansion. The volumetric deformation has hence been accounted for the existence of two different coefficients of thermal expansion, but not due to heat flux. It also justifies the removal of the inward heat flux term, from the heat flux boundary condition.

A typical thickness $\left(t_{1}+t_{2}\right)$ for a Nafion based IPMC would be $200 \mu \mathrm{m}$ and the corresponding cation layer thickness would be $20 \mu \mathrm{m}$ [10]. Therefore it is possible to select a set of values for $h, T, T_{a m b} \alpha_{y} \alpha_{z} E_{y} E_{z} v_{1}$ and $v_{z}$ to tally the deflections of the FEM coupled structural and thermal multi-physics model to the real deflections shown by an IPMC beam under a corresponding voltage. 
The advantage of this approach is that the above model parameters can then be used to simulate the deflections of any odd shaped IPMC, prior to its actual construction.

\section{Results and Discussion}

The simulation results of the above theoretical derivations and the experimental results are presented and discussed in this section.

\subsection{Results}

The experimentally verified deflected shape of a Nafion based IPMC beam of length $L=25 \mathrm{~mm}$ and stiffness of $E I=2.8416 \times 10^{-6} \mathrm{Nmm}^{2}$ with lowest (0V) and highest (1.33V) applied voltages are shown in Figs. 06 (a) and (b) respectively. The FEM simulation model of beam with same dimensions is shown in Fig. 06 (c). The parameters identified in the bi-metallic strip model of the thermal analogy are fine tuned to match the deflections shown by the FEM model with that of the actual deflections. The comparison is shown in Fig. 06 (d). A well agreement between experimental deflections and model deflections is reached throughout the length of the beam for the entire voltage range concerned by fine-tuning the model parameters of the FEM model.

A more complex soft actuator of semi circular shape is considered next. Except for the geometric shape, all other parameters such as coefficients of thermal expansion, temperatures corresponding to voltages, etc used in constructing the model are as same as those found by fine tuning FEM model with the experimental results in the beam model case discussed previously. Its deformed shape is shown in Fig. 07(a). The deformed shape obtained from the FEM simulations is shown in Fig. 07(b).

Experimental deflections of the actual semicircular complex shaped soft actuator and the simulated deflections obtained by the FEM model of the same, are compared as in Fig. 07(c).

\subsection{Discussion}

As was shown in Fig. 06(a), the IPMC material developed locally using novel cost effective fabrication technologies, is firstly cut into the strip shape and used as a cantilevered beam.
Secondly the voltage versus deflection relations of the experimental IPMC cantilevered beam are used to tune the parameters of the FEM model (Fig. 06(b)) of the beam prepared according to the same dimensions as the actual IPMC (Fig. 06(c)). The tuned parameters are then be used to predict the deformed shape of any other complex shaped IPMC actuator at the same voltage. This scenario is shown in Fig. 07. The derived modeling technique is very useful in designing complex shaped IPMC soft sensors and soft actuators.

As can be seen in Fig. 07(c), the FEM model derives the same deflected shape as the actual actuator. Hence this method of:

1. Use of large deflection bending model

2. Using bi-metallic strip analogy to model the bending action

3. Use to experimental IPMC beam model data to tune the FEM model

can be used to derive the deflected shape of any complex shaped IPMC soft actuator, which has been verified experimentally using a reasonably complicated shape soft actuator.

\section{Conclusions}

This paper uses cantilevered flexible beam model undergoing large deflections under a point load at the free end to model an IPMC Soft Sensor. The same cantilevered flexible beam model but under a uniformly distributed load undergoing large deflections is used to model an IPMC Soft Actuator. The principle of thermal expansion and contraction of a bi- metallic strip is used model the motion action in both cases.

The structural and thermal multi-physics models, used to derive the deformations of complicated shapes of actuators are implemented for simulations using finite element methods. The simulations are verified experimentally using actual IPMC materials manufactured locally. 


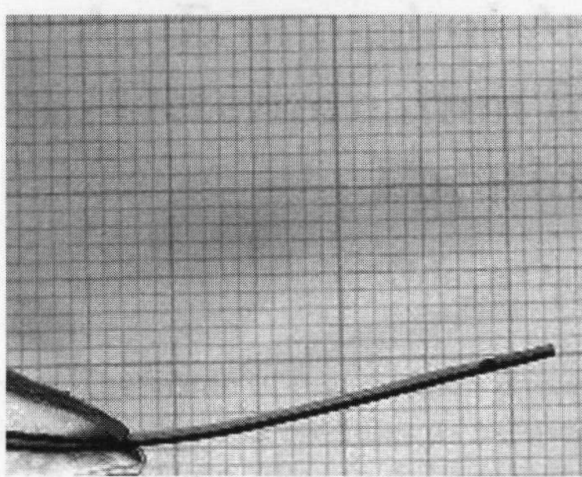

(a)

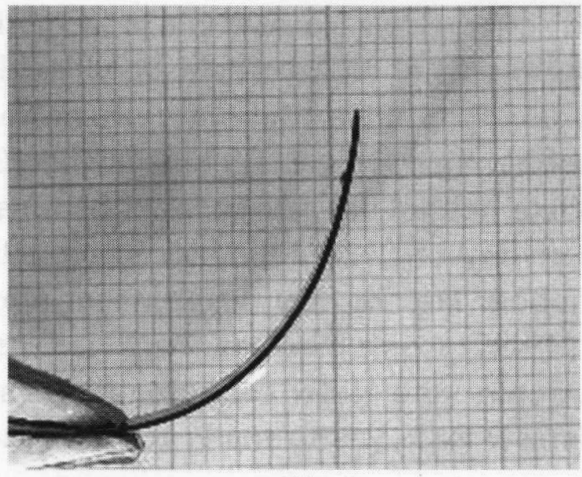

(b)

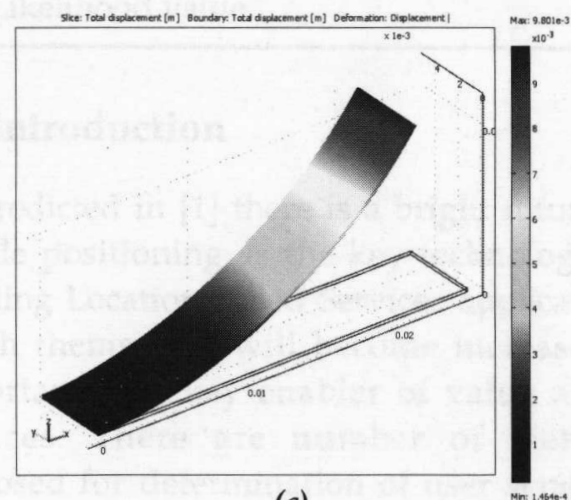

(c)

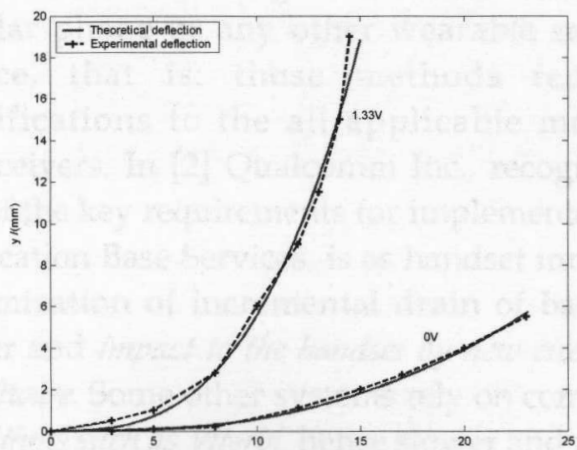

(d)

Figure 6: (a).IPMC beam deflection with lowest voltage (0V). (b) IPMC beam deflection with highest voltage (1.33V). (c) FEM model of the beam. (d)

Model tuning until it matches with actual deflections. The IPMC local fabrication process is pending patents, therefore it is not included here.

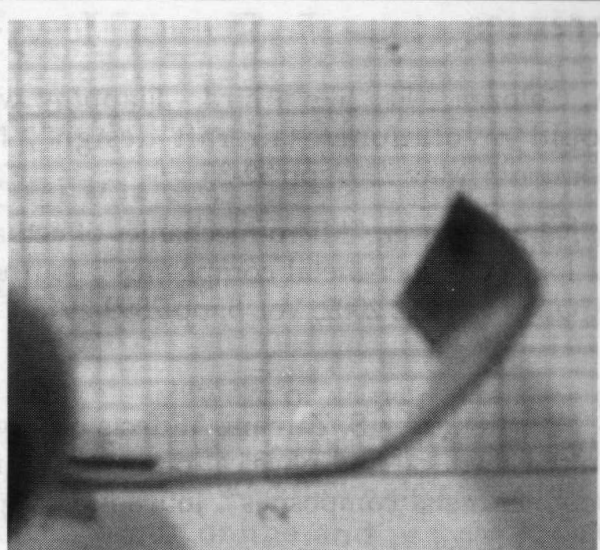

(a)

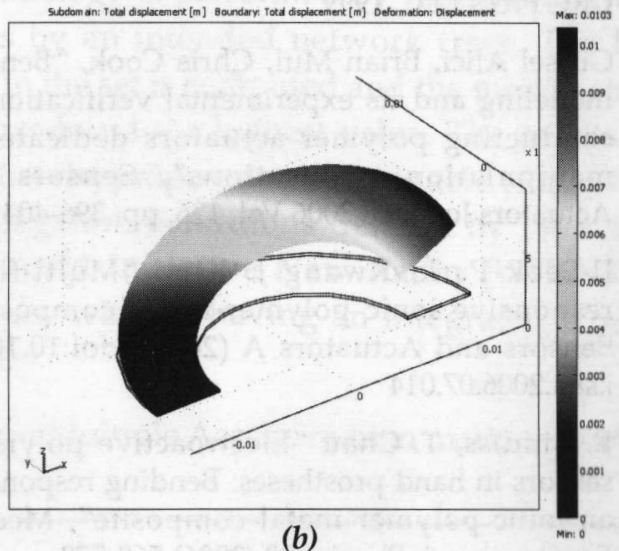

(b)

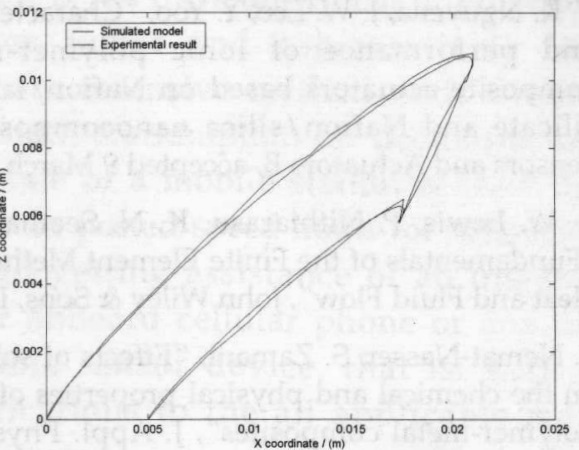

(c)

Figure 7: Experimental model verification using a complex semicircular shaped IPMC soft actuator: (a) Semi circular shaped IPMC soft actuator, (b) FEM model of the semi-circular complex shaped soft actuator, (c) Comparison of actual IPMC experimental deflection and the FEM model simulation deflection of the semi-circular complex shaped soft actuator. 


\section{References}

1. A, Colozza, "Fly like a bird - Flapping wings could revolutionize aircraft design", IEEE Spectrum, May 2007, pp 32-37.

2. S. Nemat-Nasser, "Micro-mechanics of actuation of ionic polymer-metal composites", Journal of Applied Physics 2002, Vol. 5, pp 2899-2915.

3. S. Nemat-Nasser, S. Zamani, "Effects of solvents on the chemical and physical properties of ionic polymer-metal composites", Journal of Applied Physics 2006.

4. D. G. Fertis, "Nonlinear Mechanics", 2nd Ed., CRC Press LLC, 1999.

5. Gursel Alici, Brian Mui, Chris Cook, "Bending modeling and its experimental verification for conducting polymer actuators dedicated to manipulation applications", Sensors and Actuators Journal, 2006 Vol. 126, pp 396-404.

6. Il-Seok Park, Kwang J. Kim, "Multi-fields responsive ionic polymer-metal composite", Sensors and Actuators A (2006), doi:10.1016/ j.sna.2006.07.014

7. E. Biddiss, T. Chau "Electroactive polymeric sensors in hand prostheses: Bending response of an ionic polymer metal composite", Medical Engineering \& Physics 28 (2006) 568-578.

8. V. K. Nguyena, J. W. Lee, Y. Yoo, "Characteristics and performance of ionic polymer-metal composite actuators based on Nafion/layered silicate and Nafion/silica nanocomposites", Sensors and Actuators B, accepted 9 March 2006.

9. R. W. Lewis, P. Nithiarasu, K. N. Seetharamu, "Fundamentals of the Finite Element Method for Heat and Fluid Flow", John Wiley \& Sons, Ltd.

10. S. Nemat-Nasser, S. Zamani, "Effects of solvents on the chemical and physical properties of ionic polymer-metal composites", J. Appl. Phys. 99 , (1049021 ) 2006. 